\title{
Fuzzy Boundary Control of 2D Burgers Equation with an Observer
}

\author{
Mehmet Önder Efe
}

\begin{abstract}
Fuzzy boundary control of 2D Burgers Equation is considered in this paper. The Burgers equation has extensively been considered as a benchmark problem by flow control researchers. The flavor of the problem is its multidimensionality and nonlinearity, which are the reasons making the governing equation a good start for developing a well-defined understanding of control of flows. This paper demonstrates that an infinite dimensional observer can be designed, and by the aid of the observer, a simple fuzzy boundary feedback control can be achieved under severe noise and disturbance scenarios. The results are in good compliance with the theoretical claims and the simplicity of the controller is another prominent feature.
\end{abstract}

\section{INTRODUCTION}

Due to its nonlinearity and multidimensionality, 2D Burgers equation constitutes a good benchmark among infinite dimensional systems that can enjoy the standard techniques of classical control theory. Various kinds of Burgers equation have been studied in the past. In (Donea and Huerta 2003, McDonough and Huang 2004, Efe 2004, Blender 1991, Boules and Eick 2003, Hataue 1998, Nishinari et al. 2001), a simplified version of Navier-Stokes equations given by the Partial Differential Equation (PDE) set $\underline{u}_{t}+\epsilon(\underline{u} \cdot \nabla) \underline{u}=$ $\mu \nabla^{2} \underline{u}$ with $\underline{u}$ being 2-by-1 vector function is described as the $2 \mathrm{D}$ Burgers equation. The 2D Burgers equation is therefore considered as a turbulence free cartoon for Navier-Stokes equations and has been studied in the past for modelling traffic flows, shock waves and acoustic transmission. Blender postulates a method to obtain the solution of the above mentioned PDE set iteratively, (Blender 1991). Efe demonstrates the low dimensional modelling with proper orthogonal decomposition in (Efe 2004), and Boules and Eick (2003) perform the model reduction with Fourier expansions. In (Sirendaoreji 1999, Hietarinta 2000, Zhu 1996), some other variants of $2 \mathrm{D}$ Burgers equation have been considered with the goal of finding exact solutions under certain circumstances. These types are $\left(u_{t}+u u_{x}-u_{x x}\right)_{x}+u_{y y}=0$ in (Sirendaoreji 1999, Hietarinta 2000), $u_{t}+u u_{x}+u_{x x}+$ $u_{x x x}=0$ in (Zhu 1996). Nishinari et al. (2001) focus on cellular automaton, which is extensively studied for developing models of traffic flow, fluids and immune systems, and therefore a good model to work on is a variant of Burgers equation. In Hataue (1998), the dynamics that arises upon discretization of 2D Burgers equation is analyzed. The effects of chosen time step $(\Delta t)$ for getting physically reasonable numerical solutions are elaborated. Wescott et al. (2001) present a computational technique to obtain the numerical solutions of PDEs having nonlinear convection terms like

TOBB University of Economics and Technology, Department of Electrical and Electronics Engineering, Sögütözü, Ankara, Turkey, E-mail: onderefe @ieee.org, Phone: +90-312-292-4064, Fax: +90-312-292 4091
2D Burgers equation and Navier-Stokes equations. The goal of the paper is to reduce the computation time without giving concessions from the accuracy. Boules and Eick (2003) obtain the solution of Burgers equation for a specific boundary regime and initial conditions. Using a truncated Fourier series expansion yields an autonomous ODE set, the solution of which approximates the numerical solution, and the derived model rebuilds the situation implied by the chosen initial and boundary conditions. When the 1D version given by $u_{t}=-u u_{x}+u_{x x}$ is taken into consideration, it is seen that a significant amount of research outcome has been reported on modelling and control system design, (Hinze and Volkwein 2002, Burns et al. 2002a-b, Vedantham 2000, Park and Jang 2002, Liu and Krstić 2000-2001, Krstić 1999, Efe and Özbay 2003a-2004, Efe et al. 2004). A majority of the cited references emphasizes the similar difficulties as the motivating factors and focus on the solutions and solvability issues. The current paper, on the other hand, derives an infinite dimensional observer that can be used for boundary control purposes. The contribution of this paper is the to demonstrate that simple yet effective observers can be devised to obtain the information about the infinite dimensional process, and this information can be used for boundary control purposes. The second section discusses the design of the observer, the third section presents the fuzzy controller, simulation conditions and obtained results. The concluding remarks are given at the end of the paper.

\section{DESIGN OF THE OBSERVER}

Define $\Omega:=\{(x, y) \mid(x, y) \in[0,1] \times[0,1]\}$ as the physical domain of the process and the observer described by

$$
u_{t}=-k_{1} u u_{x}-k_{2} u u_{y}+\mu u_{x x}+\mu u_{y y},
$$

$$
w_{t}=-k_{1} w w_{x}-k_{2} w w_{y}+\mu w_{x x}+\mu w_{y y}+K(u-w),
$$

respectively. In (1) and (2), $u=u(x, y, t), w=w(x, y, t)$ and $k_{1}, k_{2}$ and $\mu$ are positive valued known process parameters, $K$ is the observer gain. The boundary conditions are specified by 


$$
\begin{aligned}
u_{y}(x, 0, t) & =\beta_{0} u(x, 0, t), \\
w_{y}(x, 0, t) & =\beta_{0} w(x, 0, t), \\
u_{x}(x, 1, t) & =-\beta_{1} u(x, 1, t), \\
w_{y}(x, 1, t) & =-\beta_{1} w(x, 1, t), \\
u_{x}(0, y, t) & =\zeta_{0} u(0, y, t), \\
w_{x}(0, y, t) & =\zeta_{0} w(0, y, t), \\
u_{x}(1, y, t) & =-\zeta_{1} u(1, y, t), \\
w_{x}(1, y, t) & =-\zeta_{1} w(1, y, t),
\end{aligned}
$$

where $\zeta_{0}, \zeta_{1}, \beta_{0}$ and $\beta_{1}$ are positive numbers. The problem is to reconstruct $u(x, y, t)$ over $\Omega$ by designing an observer, whose variable is $w(x, y, t)$, and to perform observer based feedback control through boundary (corner) excitations.

Theorem 2.1: For the 2D Burgers equation given in (1), an infinite dimensional observer having the structure given by (2) can be designed if and only if there exists a $\lambda>0$ such that $\left|k_{1} \frac{\partial\left(u^{2}-w^{2}\right)}{\partial x}+k_{2} \frac{\partial\left(u^{2}-w^{2}\right)}{\partial y}\right| \leq \lambda|u-w|$ is satisfied. Such an observer with a positive $K \in \Re$ results in globally exponentially stable reconstruction over $(x, y, t) \in[0,1] \times$ $[0,1] \times[0, \infty)$.

Proof: Consider the Lyapunov function candidate

$$
V=\frac{1}{2} \int_{0}^{1} \int_{0}^{1}(u(x, y, t)-w(x, y, t))^{2} d x d y .
$$

For the sake of the simplicity, we drop the arguments of the variables $u$ and $w$, and take the time derivative of the Lyapunov function candidate above. This yields

$$
\begin{aligned}
\dot{V}= & \int_{0}^{1} \int_{0}^{1}(u-w)\left(u_{t}-w_{t}\right) d x d y \\
= & \int_{0}^{1} \int_{0}^{1}(u-w)\left(-k_{1} u u_{x}-k_{2} u u_{y}+\mu u_{x x}\right. \\
& +\mu u_{y y}+k_{1} w w_{x}+k_{2} w w_{y}-\mu w_{x x}-\mu w_{y y} \\
& -K(u-w)) d x d y \\
= & -2 K V+\int_{0}^{1} \int_{0}^{1}(u-w)\left(-k_{1} u u_{x}-k_{2} u u_{y}\right. \\
& +\mu u_{x x}+\mu u_{y y}+k_{1} w w_{x}+k_{2} w w_{y}-\mu w_{x x} \\
& \left.-\mu w_{y y}\right) d x d y .
\end{aligned}
$$

Set $s:=u-w$, we have

$$
\begin{aligned}
& \dot{V}=-2 K V+\mu \int_{0}^{1} \int_{0}^{1}\left(s s_{x x}+s s_{y y}\right) d x d y- \\
& \frac{1}{2} \int_{0}^{1} \int_{0}^{1}(u-w) \star \\
& \left(k_{1} \frac{\partial\left(u^{2}-w^{2}\right)}{\partial x}+k_{2} \frac{\partial\left(u^{2}-w^{2}\right)}{\partial y}\right) d x d y
\end{aligned}
$$

Using the boundary conditions in (3), it is straightforward to show that the following equality holds true:

$$
\begin{aligned}
& \int_{0}^{1} \int_{0}^{1} s s_{x x} d x d y=\int_{0}^{1} s(1, y) s_{x}(1, y) \\
& \left.-s(0, y) s_{x}(0, y)\right) d y-\int_{0}^{1} \int_{0}^{1} s_{x}^{2} d x d y \\
& =-\int_{0}^{1} \zeta_{1} s(1, y)^{2}+\zeta_{0} s(0, y)^{2} d y-\int_{0}^{1} \int_{0}^{1} s_{x}^{2} d x d y .
\end{aligned}
$$

Likewise we have,

$$
\begin{aligned}
& \int_{0}^{1} \int_{0}^{1} s s_{y y} d x d y=\int_{0}^{1} s(x, 1) s_{y}(x, 1)- \\
& \left.s(x, 0) s_{y}(x, 0)\right) d x-\int_{0}^{1} \int_{0}^{1} s_{y}^{2} d x d y \\
& =-\int_{0}^{1} \beta_{1} s(x, 1)^{2}+\beta_{0} s(x, 0)^{2} d x \\
& -\int_{0}^{1} \int_{0}^{1} s_{y}^{2} d x d y .
\end{aligned}
$$

Utilizing these results with the assumption of the theorem lets us rewrite (6) as follows:

$$
\begin{aligned}
& \dot{V}=-2 K V-\mu \int_{0}^{1} \zeta_{1} s(1, y)^{2}+\zeta_{0} s(0, y)^{2} d y \\
& -\mu \int_{0}^{1} \beta_{1} s(x, 1)^{2}+\beta_{0} s(x, 0)^{2} d x \\
& -\mu \int_{0}^{1} \int_{0}^{1}\left(s_{x}^{2}+s_{y}^{2}\right) d x d y-\frac{1}{2} \int_{0}^{1} \int_{0}^{1}(u-w) \star \\
& \left(k_{1} \frac{\partial\left(u^{2}-w^{2}\right)}{\partial x}+k_{2} \frac{\partial\left(u^{2}-w^{2}\right)}{\partial y}\right) d x d y \\
& \leq-2 K V-\mu \int_{0}^{1} \zeta_{1} s(1, y)^{2}+\zeta_{0} s(0, y)^{2} d y \\
& -\mu \int_{0}^{1} \beta_{1} s(x, 1)^{2}+\beta_{0} s(x, 0)^{2} d x \\
& -\mu \int_{0}^{1} \int_{0}^{1}\left(s_{x}^{2}+s_{y}^{2}\right) d x d y+\frac{1}{2} \int_{0}^{1} \int_{0}^{1}|u-w| \star \\
& \left|k_{1} \frac{\partial\left(u^{2}-w^{2}\right)}{\partial x}+k_{2} \frac{\partial\left(u^{2}-w^{2}\right)}{\partial y}\right| d x d y
\end{aligned}
$$




$$
\begin{aligned}
& \leq-2 K V-\mu \int_{0}^{1} \zeta_{1} s(1, y)^{2}+\zeta_{0} s(0, y)^{2} d y \\
& -\mu \int_{0}^{1} \beta_{1} s(x, 1)^{2}+\beta_{0} s(x, 0)^{2} d x \\
& -\mu \int_{0}^{1} \int_{0}^{1}\left(s_{x}^{2}+s_{y}^{2}\right) d x d y \\
& +\frac{1}{2} \int_{0}^{1} \int_{0}^{1} \lambda|u-w|^{2} d x d y \\
& =-(2 K-\lambda) V-\mu \int_{0}^{1} \zeta_{1} s(1, y)^{2}+\zeta_{0} s(0, y)^{2} d y \\
& -\mu \int_{0}^{1} \beta_{1} s(x, 1)^{2}+\beta_{0} s(x, 0)^{2} d x \\
& -\mu \int_{0}^{1} \int_{0}^{1}\left(s_{x}^{2}+s_{y}^{2}\right) d x d y \\
& <0 .
\end{aligned}
$$

This results shows that the state of the nonlinear process in (1) can be reconstructed by the nonlinear observer in (2). The next section presents the practical implications of the theorem and its proof above.

\section{FuZZY BOUNDARY CONTROL OF THE SySTEM}

\section{A. Fuzzy Controller}

Fuzzy systems are well known for their rule based structure letting the designer compose local decisions of a possibly complicated map. The map in this context refers to the control law. Although many variants of fuzzy controllers are available, (Jang et al, 1997), we aim to demonstrate how feasible the use of fuzzy controllers in the control of spatially continuous processes. For this purpose, we adopt the following fuzzy model with triangular membership functions

$$
\gamma_{00}=\frac{\sum_{i=1}^{R} y_{i} \Psi_{i}}{\sum_{i=1}^{R} \Psi_{i}}
$$

where $\gamma_{00}$ is the controller output, $R$ is the number of rules contained in the rule base, $y_{i}$ is the defuzzifier parameter associated with the $\mathrm{i}$-th rule, $\Psi_{i}$ is the firing strength of $\mathrm{i}$-th rule calculated as given below:

$$
\Psi_{i}=\mu_{Z_{i}^{i e}, e_{1}} \star \mu_{Z_{i}^{e}, e_{2}}
$$

where $e_{1}(t)=\int_{0}^{t} e(\sigma) d \sigma$ and $e_{2}(t)=e(t)$ with $e(t)$ being an error measure yet to be defined. The generic form of the i-th rule is

IF $e_{1}$ is $Z_{i}^{i e}$ AND $e_{2}$ is $Z_{i}^{e}$ THEN $\gamma_{00}$ is $y_{i}$

In the above rule structure, $Z_{i}^{i e}$ and $Z_{i}^{e}$ denote the fuzzy sets corresponding to the integral of the error and the error respectively, and $y_{i}$ is the conclusion value (the defuzzifier parameter) for the i-th rule. The membership functions are described in (13), and the defuzzifier parameters are tabulated in Table 1 with the nine rules.

$$
\begin{aligned}
\mu_{\mathcal{N}, e_{1}} & =\max \left(0, \min \left(1,-e_{1} / L_{i e}\right)\right) ; \\
\mu_{\mathcal{Z}, e_{1}} & =\max \left(0, \min \left(e_{1} / L_{i e}+1,-e_{1} / L_{i e}+1\right)\right) ; \\
\mu_{\mathcal{P}, e_{1}} & =\max \left(0, \min \left(1, e_{1} / L_{i e}\right)\right) ; \\
\mu_{\mathcal{N}, e_{2}} & =\max \left(0, \min \left(1,-e_{2} / L_{e}\right)\right) ; \\
\mu_{\mathcal{Z}, e_{2}} & =\max \left(0, \min \left(e_{2} / L_{e}+1,-e_{2} / L_{e}+1\right)\right) ; \\
\mu_{\mathcal{P}, e_{2}} & =\max \left(0, \min \left(1, e_{2} / L_{e}\right)\right) ;
\end{aligned}
$$

where $L_{e}=1$ and $L_{i e}=0.8$ are the user-specified membership function parameters.

TABLE I

The Defuzzifier Parameters

\begin{tabular}{|c|c|c|c|}
\hline \hline & $e_{1}$ is $\mathcal{N}$ & $e$ is $\mathcal{Z}$ & $e$ is $\mathcal{P}$ \\
\hline \hline$e_{2}$ is $\mathcal{N}$ & -80 & -1 & 78 \\
\hline$e_{2}$ is $\mathcal{Z}$ & -79 & 0 & 79 \\
\hline$e_{2}$ is $\mathcal{P}$ & -78 & 1 & 80 \\
\hline
\end{tabular}

\section{B. Use of Fuzzy Controller}

In this part, we consider the observer based boundary control of the system in (1). For this purpose, we choose $(x, y)=(0,0)$ corner as the entry for control excitation denoted by $\gamma_{00}(t)$, and other three corners as the entries for disturbances, i.e. $\gamma_{01}(t), \gamma_{10}(t)$ and $\gamma_{11}(t)$. Once the corner conditions are specified, the numerical solution for the boundaries of the domain $\Omega$ are obtained. For example, we solve the equation set

$$
\begin{aligned}
u_{t} & =-k_{1} u u_{x}+\mu u_{x x}, \\
w_{t} & =-k_{1} w w_{x}+\mu w_{x x}+K(u-w)
\end{aligned}
$$

along $x=0$ and $x=1$ segments. A similar strategy is followed for other two segments of the boundary. The numerical solution is obtained by using Crank-Nicholson implicit method, (Farlow 1993). The boundary conditions in (3) are forced and the interior grid solution of the PDE set in (1)-(2) are obtained. The settings of the simulation are tabulated in Table 2.

TABLE II

Simulation SetTings

\begin{tabular}{|c|c|}
\hline \hline$k_{1}$ & 5 \\
\hline$k_{2}$ & 5 \\
\hline$\mu$ & 10 \\
\hline$\zeta_{0}$ & 1 \\
\hline$\zeta_{1}$ & 1 \\
\hline$\beta_{0}$ & 1 \\
\hline$\beta_{1}$ & 1 \\
\hline$\Delta t$ & $1 \mathrm{msec}$. \\
\hline$T$ & $1 \mathrm{sec}$. \\
\hline$K$ & 10 \\
\hline$N_{x}$ & 30 \\
\hline \hline
\end{tabular}

In order to demonstrate that the observer works well under noisy observations of $u(x, y, t)$, a normally distributed measurement noise corrupts the read quantity, $u$. The controlled variable is $u\left(x_{m}, y_{m}, t\right)$ with $x_{m}$ and $y_{m}$ being the 
coordinates of the arbitrarily chosen measurement location. The control problem is to force $u\left(x_{m}, y_{m}, t\right)$ towards a reference signal $r(t)$ at $(x, y)=\left(x_{m}, y_{m}\right)$. For this purpose, we have set $\left(x_{m}, y_{m}\right)=(0.5172,0.5172)$. As the reference signal, we utilize $r(t)=\operatorname{sign}(\sin (2 \pi 2 t))$ and define $e(t)=$ $r(t)-w\left(x_{m}, y_{m}, t\right)$. This choice of the reference signal is deliberate as it excites the shock response of both the process and observer, and makes it easy to see the steady state behavior.
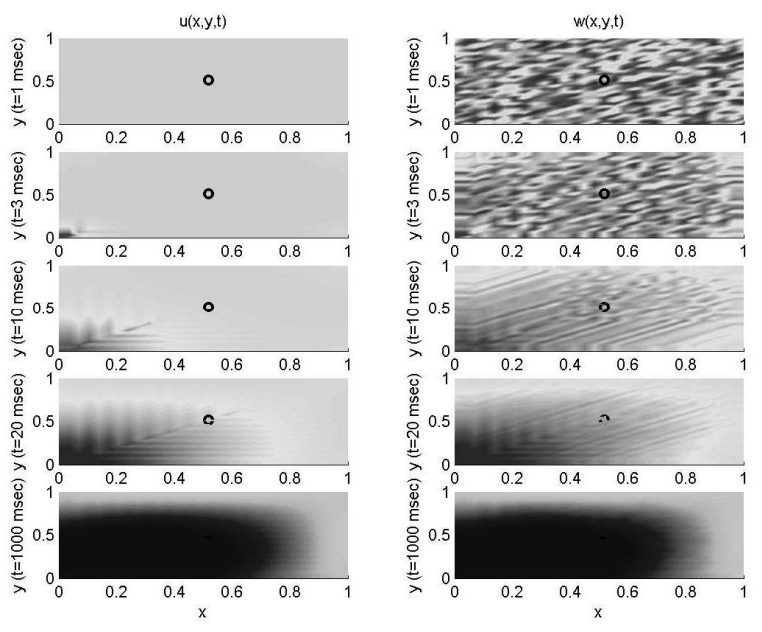

Fig. 1. Simulation results with fuzzy controller
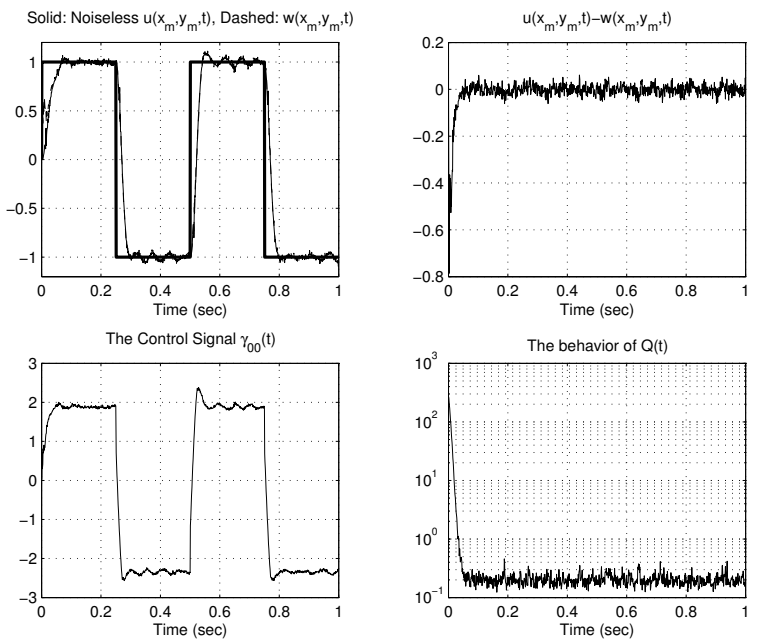

Fig. 2. Simulation results with fuzzy controller

Aside from the reference signal's step-like nature, the disturbance signals are set as

$$
\begin{aligned}
& \gamma_{01}(t)=0.1 \operatorname{sign}(\sin (2 \pi 20 t)), \\
& \gamma_{10}(t)=0.1 \operatorname{sign}(\sin (2 \pi 17 t)), \\
& \gamma_{11}(t)=0.1 \operatorname{sign}(\sin (2 \pi 15 t)),
\end{aligned}
$$

where we inject abruptly changing signals at different frequencies. This kind of a disturbance scenario lets us obtain a rich set of disturbance effect at the fixed location $\left(x_{m}, y_{m}\right)$.
A last issue to figure out is the reconstruction performance of the observer. For this purpose, we define the following integral as the measure of performance,

$$
Q(t)=\int_{0}^{1} \int_{0}^{1}(u(x, y, t)-w(x, y, t))^{2} d x d y .
$$

In Figure 1, a matrix of subplots is depicted with a circle indicating the location $\left(x_{m}, y_{m}\right)$. The plots on the left column are for $u(x, y, t)$ and those on the right column are for $w(x, y, t)$. The first four rows are the snapshots from both processes at $t=1,3,10$ and $20 \mathrm{msec}$. instants. The last row is for the final time, $T=1 \mathrm{sec}$. Clearly, the transient phase passes very quickly and a convergence is observed. This fact is further visible in the bottom right subplot of Figure 2, where the convergence is achieved after the first $50 \mathrm{msec}$. time.

Looking at the results in Figure 2, a good tracking is seen in the top left subplot, where the reference signal $r(t)$ (thick curve), $u\left(x_{m}, y_{m}, t\right)$ (solid curve) and $w\left(x_{m}, y_{m}, t\right)$ (dashed curve) are plotted together. The process variable and the observer variable are almost indistinguishable, which is a numerical evidence to the analytical claims and existence of a $\lambda>0$. In the top right subplot, this difference is shown, where the convergent behavior is noteworthy. The bottom left subplot shows the control signal $\left(\gamma_{00}(t)\right)$ that is applied from the corner $(x, y)=(0,0)$. Unsurprisingly, although we impose unit magnitude reference signal at the measurement point, the signal that needs to be applied from the corner is higher in magnitude than the reference. This fact is because the other three corners are driven by small magnitude disturbances, and the control signal magnitude needs to be larger as the measurement point gets away from the control entry, i.e. $(x, y)=(0,0)$. A natural consequence of this is the sufficiency of relatively small control effort in the close neighborhood of the control entry, and large control effort in the vicinity of locations where the solution is independently specified. For example, in the vicinity of $(x, y)=(1,1)$, the control signal must overcome the effect of corner condition, and naturally the control effort is expectedly large.

Clearly a last question in this section should ask whether it was difficult to find such a parameter set that works good. The answer is yes, after a few trials, a good parametrization of membership functions, a good resolution parameter $\left(N_{x}\right)$ and a good observer gain $(K)$ can be set. We set these values by trial and error.

\section{CONCLUSIONS}

This paper demonstrates that a nonlinear infinite dimensional observer can be designed for a nonlinear infinite dimensional process. Despite the spatial continuity, under mild assumptions, the design problem enjoys the classical approaches of control theory. A simple yet successful fuzzy boundary control is demonstrated. The results indicate that the observer reconstructs the process state in a globally exponentially stable evolution, and the closed loop control performance is satisfactory under severely corrupted 
measurements and corner disturbances. The computational advantages of the controller is another prominent feature that deserves emphasis. The contribution of this paper is the method followed to devise a nonlinear observer for a nonlinear infinite dimensional problem.

Future research aims to demonstrate such a strategy for complicated forms of Burgers equation as in (Donea and Huerta 2003, McDonough and Huang 2004, Efe 2004, Blender 1991, Boules and Eick 2003, Hataue 1998, Nishinari et al. 2001). The contribution at large is to underline the design possibility of observers and observer based controllers for flow and fluid problems.

\section{Acknowledgments}

This work was supported in part by the AFRL/VA and AFOSR through the Collaborative Center of Control Science at the Ohio State University (Contract F33615-01-2-3154).

The author would like to thank Prof. H. Özbay, Prof. Mo Samimy, Dr. J.H. Myatt, Dr. J. DeBonis, Dr. R.C. Camphouse, Dr. P. Yan, X. Yuan and E. Caraballo for fruitful discussions in devising the presented work.

\section{REFERENCES}

[1] Aström, K.J. and Hägglund, T.H., 1995, PID Controllers: Theory, Design and Tuning. ISA - The Instrumentation, Systems, and Automation Society, North Carolina, pp.111.

[2] Blender, R., 1991, "Iterative solution of nonlinear partialdifferential equations," Journal of Physics A: Mathematical and General. 24, 10, L509-L512.

[3] Boules, A.N. and Eick, I.J., 2003, "A spectral approximation of the two-dimensional Burgers equation," Indian Journal of Pure \& Applied Mathematics. 34, 2, pp.299-309.

[4] Burns, J.A., King, B.B. and Zietsman, L., 2002a, "On the computation of singular functional gains for linear quadratic optimal boundary control problems," Proc. of the 3rd Theoretical Fluid Mechanics Meeting, St. Louis, USA, June 24-26, AIAA 2002-3074.

[5] Burns, J.A., King, B.B., Rubio, A.D. and Zietsman, L., 2002b, "Functional gain computations for feedback control of a thermal fluid," Proc. of the 3rd Theoretical Fluid Mechanics Meeting, St. Louis, USA, June 24-26, AIAA 2002-2992.

[6] Donea, J. and Huerta, A., 2003, Finite Element Methods for Flow Problems John Wiley \& Sons, West Sussex, pp.252-253.

[7] Efe, M.Ö., 2004, A Numerical Approach to Reduced Order Modeling of Nonlinear and Multidimensional PDEs. European Journal of Automatic Control, (submitted for publication).

[8] Efe, M.Ö. and Özbay, H., 2003a, "Integral action based Dirichlét boundary control of Burgers equation," Proc. of the IEEE Int. Conf. on Control Applications, (CCA'2003), Istanbul, Turkey, June 23-25, pp.1267-1272.

[9] Efe, M.Ö., Yuan, X., Özbay, H. and Samimy, M., 2004, "Interpolating local models of POD using fuzzy decision mechanisms," 9th Mechatronics Forum International Conference, Aug. 30 - Sep. 1, Ankara, Turkey, pp.347-356.

[10] Efe, M.Ö. and Özbay, H., 2004, "Low dimensional modeling and Dirichlét boundary controller design for Burgers equation," International Journal of Control, 77, 10, pp.895-906.

[11] Farlow, S.J., 1993, Partial Differential Equations for Scientists and Engineers. Dover Publications Inc., New York, pp.317322 .
[12] Hataue, I., 1998, "Mathematical and numerical analyses of dynamical structure of numerical solutions of two-dimensional fluid equations," Journal of the Physical Society of Japan. 67, 6, 1895-1911.

[13] Hietarinta, L., 2000, "Comments on "Exact solutions of the two-dimensional Burgers equation', Journal of Physics A: Mathematical and General. 33, 5157-5158.

[14] Hinze, M. and Volkwein, S., 2002, "Analysis of instantaneous control for Burgers equation," Nonlinear Analysis. 50, 1-26.

[15] Jang, J.-S.R., Sun, C.-T. and Mizutani, E., 1997, Neuro-Fuzzy and Soft Computing, Prentice Hall, Upper Saddle River, NJ.

[16] Krstić, M., 1999, "On global stabilization of Burgers equation by boundary control," Systems and Control Letters. 37, 123141.

[17] Liu, W.-J. and Krstić, M., 2000, "Backstepping boundary control of Burgers equation with actuator dynamics," Systems and Control Letters. 41, 291-303.

[18] Liu, W.-J. and Krstić, M., 2001, "Adaptive control of Burgers equation with unknown viscosity," Int. Journal Adaptive Control and Signal Processing. 15, 745-766.

[19] McDonough, J.M. and Huang, M.T., 2004, “A 'poor man's NavierStokes equation' derivation and numerical experimentsthe 2-D case," International Journal for Numerical Methods in Fluids. 44, 545578.

[20] Nishinari, K., Matsukidaira, J. and Takahashi, D., 2001, "Twodimensional Burgers cellular automaton," Journal of the Physical Society of Japan. 70, 8, 2267-2272.

[21] Park, H.M. and Jang, Y.D., 2002, "Control of Burgers equation by means of mode reduction," Int. Journal of Engineering Science. 38, 785-805.

[22] Sirendaoreji, S.J., 1999, "Exact solutions of the twodimensional Burgers equation," Journal of Physics A: Mathematical and General. 32, 6897-6900.

[23] Vedantham, R., 2000, "Optimal control of the viscous Burgers equation using an equivalent index method," Journal of Global Optimization. 18, 255-263.

[24] Wescott, B.L., Rizwan-uddin, 2001, "An efficient formulation of the modified nodal integral method and application to the two-dimensional Burgers equation," Nuclear Science and Engineering. 139, 3, 293-305.

[25] Zhu, Z., 1996, "Exact solutions for a two-dimensional KdVBurgers-type equation," Chinese Journal of Physics, 34, 4, 1101-1105. 